\title{
Image Enhancement based on Fusion using 2D LPDCT and Modified PCA
}

\author{
C. Rama Mohan, S. Kiran, Vasudeva, A. Ashok Kumar
}

\begin{abstract}
The images play a vital role in various fields of applications; medical field is the one, where images more widely used in diagnosis. Best image data analysis results if the quality of the image is high. To attain best image quality some popular techniques are available, among that image fusion is one of the technique, it enhances the information of the image by selecting and merging the significant information from two or more similar multi-focus images. Using the features of image fusion a new technique is proposed in this paper. In proposed technique, fusion of sources images with 2D Laplacian Pyramid Discrete Cosine Transformation (2D LP - DCT) and Modified Principal Component Analysis (MPCA). In this, two similar multi-focus images are considered, first, they undergone to $2 D$ LP-DCT and then MPCA technique. The $2 D$ LP-DCT enhances important image features, which are best utilized in image fusion and results good image quality. In Modified PCA, the concept of dimensionality reduction is used. The experimental results indicate that the suggested strategy can produce fused images with good visual quality and computational effectiveness than other state-of-the-art works.
\end{abstract}

Keywords: Laplacian Pyramid, Image Fusion, Multi-focus Images, DCT, Quality Evaluation Metrics, Image Quality, Modified Principal Component Analysis

\section{INTRODUCTION}

Fusion of images is a procedure in which fusion rules are used to combine two or more images from the same scene with different focus values. The fusion results in an informative and composite image that is more suitable for visual perception, object detection, and target recognition [1-5]. The goal of image fusion is to integrate additional and redundant information from multiple images to create more composite images than any source image. Image fusion is widely used to apply distinct types of information in their entirety. Due to the combination of information with different features, mixed pictures can provide a better understanding and a more reliable outcome. The fusion method can increase the efficiency of subsequent processing operations such as extraction and identification of objects and segmentation.

An algorithm for image fusion seeks primarily to reduce the number of data errors, but also to combine significant visual details from various images so that the image results in exact and detailed information without introducing objects. A wider view of the objects observed may be

Revised Manuscript Received on 14 August, 2019.

C. Rama Mohan, Research Scholar, Department of CSE, VTU, Belgaum 590018, Karnataka, India (Email: ramamohanchinnem@gmail.com)

S. Kiran, Department of CSE, YSR Engineering College of YVU, Proddatur 516360, A. P., India(Email: rkirans125@gmail.com)

Vasudeva, Department of CSE, SMVITM, Bantakal, Udupi - 574115 , Karnataka, India.(Email: vasudeva.cs@ sode-edu.in)

A. Ashok Kumar, Department of Physics, YSR Engineering College of YVU, Proddatur 516360, A. P., India(Email: drashok.yvuce@gmail.com) provided by spectral, spatial and temporal image resolution [6]. Multi-source image information may be gathered by fast technological progress to create a mixture between space and spectra information of high quality images [7-8]. Fusion involves a wide variety of medical, surveillance, military, microscopic, remote sensing systems, computer vision, robotic vision, robotic vision, and navigation.

The processing level, such as the pixel level, the signal level and the function level-based on the image fusion-can generally be used. The traditional image fusion schemes performed fusion on source images, often with serious side effects such as contraster reduction. Additional scientists have recognized the need for fusion as mathematical change in the field of transformation [9]. In many applications, wavelets transformation was used like restoring, removing noise in a image, improving images on the edge and extracting the features; the two-dimensional data found in the pictures were not easily captured with wavelets [10]. Different techniques for transformation were suggested for the image signals that incorporate direction and multiresolution so that borders could not be captured efficiently in natural images. Several transformation techniques were proposed for image signals with directional and multiresolution signals that could therefore not capture natural photographs efficiently. Good image performance such as $\mathrm{Q}^{\mathrm{AB} / \mathrm{F}}, \mathrm{QW}, \mathrm{QS}, \mathrm{QE}, \mathrm{QC}, \mathrm{QCB}, \mathrm{CQM}, \mathrm{QG}, \mathrm{QM}, \mathrm{QP}, \mathrm{H}$, SD, SF, RMSE, PFE, MAE, SNR, PSNR, MSE, and PSNR_HVSM cannot be retrieved using the simplistic primitive technique.

Several investigators have suggested a range of image fusion algorithms based on the combination between the DWT and PCA, morphologies and morphological techniques [11-14]. These methods are significantly better than the average simple, maximum, and minimum [15].

A number of papers have been published late on the development of various pixel image fusion algorithms. Many algorithms are based on transform wavelets [ 16-17 ], pyramids transform [ 18 ], statistic signal processing [ 19 ], principal component analysis [ 16, 19, 29 ], fuzzy logic [ 20, 25 ], DCT [ 15, 21-22 ] portioning [ 23, 28 ], etc. DCT has been shown to be well for image compression and is well received in several applications of image preprocessing. The domain-based image fusion technique for Discrete Cosine Transform (DCT) has demonstrated that it has proven more convenient and time-saving in real-time images or video systems[16].

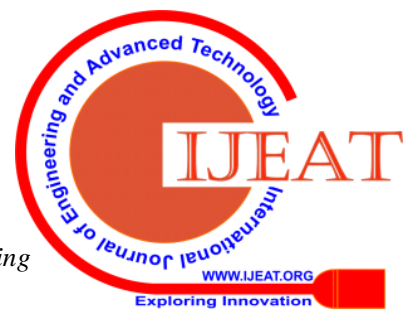


This paper is designed to fuse multifocus images [32] with the 2D laplacian pyramid discrete cosine transform and fused image with modified principal component analysis. The fuse image extraction feature is determined with different parametric analysis. The suggested technique is contrasted with approved techniques of fusion such as SWT, MSVD, NSCT, CV, DTCWT, RP, and LP. The result of the 2D LPDCT and MPCA system shows that statistical parameters are substantially better than other methods already in use.

\section{DISCRETE COSINE TRANSFORM (DCT)}

In the field of image processing, Discrete Cosine Transform is widely used and accepted. DCT's coefficients focus on the region of low frequency. High frequency coefficients contribute to excellent energy compactness and edges. DCT uses cosine waves that adjust the image data. DCT coefficients are encoded independently after the decorrelation process without losing compression efficiency. Compared with DFT, DCT is purely real and has numerous science and engineering applications. DCT technique employs spectral methods for partial differential equation numerical solutions.

\section{Limitations of Discrete Cosine Transform}

- Higher spectral coefficients cause the transformation of DCT to blur.

- Some low spectral coefficients cause graininess in smooth parts of the image in the case of quantization.

- Block boundaries consist of series blocking DCT artifacts as each block is encoded with different strategies and external quantization.

\section{PROPOSED METHOD}

The proposed method includes two processes: the 2D LPDCT - based image fusion process and MPCA process, shows the proposed method framework. The following are the two processes.

\section{1. 2D Laplacian Pyramid Based Image Fusion}

The image fusion based on the 2D LP-DCT [ 26 - 27 ] is described in Algorithm 1, applicable to fusion of first and second image features with a level ' $\mathrm{r}$ '. The description of some important techniques and algorithms in detail is as follows.

\footnotetext{
Algorithm 1. 2D LP-DCT Based Image Fusion

Input : Multifocus images.

Step 1 : Load the multifocus images from the source.

Step 2 : Perform the 2D LP-DCT on multifocus input images.

Step 3 : Fuse components of input images via the following guidelines for obtaining composites of input images.

Step 4 : Perform inverse DCT on the composite frequency components to obtain the fused

image.

Output: Fused image.
}

The method for the construction and restoration of the Laplacian pyramid is outlined below. The image $f(x, y)$ of the size $\mathrm{MxN}$ is divided into rows and concatenates these rows to form $1 D$ vector data $f(x)$ the size of which would be MN. The Matlab code for the following is:

function $[\mathrm{P}]=$ conversion_2D_array_to_1D_vector $(\mathrm{P}, \mathrm{M}$, N)

$\mathrm{P}(2: 2:$ end,$:)=\mathrm{P}(2: 2:$ end,end: $-1: 1)$

$\mathrm{P}=\operatorname{reshape}\left(\mathrm{P}^{\prime}, 1, \mathrm{M}^{*} \mathrm{~N}\right)$

By reversing the operation, the $2 \mathrm{D}$ image can be reconstructed from 1D vector data and the correct Matlab code is:$$
\text { function }[\mathrm{P}]=\text { conversion_1D_vector_to_2D_array }(\mathrm{P}, \mathrm{M} \text {, }
$$
N)

$\mathrm{P}=\operatorname{reshape}(\mathrm{P}, \mathrm{N}, \mathrm{M})^{\prime}$

$\mathrm{P}(2: 2:$ end,: $)=\mathrm{P}(2: 2:$ end,end: $-1: 1)$

Likewise, the image $f(x, y)$ of the $\mathrm{MxN}$-size is broken into columns and these columns are concatenated to form a 1D-vector $\mathrm{f}(\mathrm{x})$ with a $\mathrm{MN}$ dimension. Matlab code is: $\mathrm{P}=$ $2 \mathrm{D}$ array to $1 \mathrm{D}$ vector $\left(\mathrm{P}^{\prime}, \mathrm{M}, \mathrm{N}\right)$; at level $0 \mathrm{f} 0 \mathrm{MxN}$ sizes, the image is lowered to the next level $0.5 \mathrm{Mx} 0.5 \mathrm{~N} \mathrm{f1}$, both of which is spatial density and resolution. The version of $\mathrm{fl}$ and so on is also reduced to f2. Reduced image is obtained by taking DCT and applying the IDCT in both directions to the first half of the coefficients. The image reduction level to level is performed with the reduction function $\mathrm{P}$.

Reduction Function P :

$\mathrm{f}_{\mathrm{r}}=\mathrm{P}\left(\mathrm{f}_{\mathrm{r}-1}\right)$

The opposite of the reduction function is the extension function $\mathrm{E}$. This result is to expand the image of $\mathrm{MxN}$ size into a $2 \mathrm{Mx} 2 \mathrm{~N}$ image by taking IDCT in vertical direction following the padding of $\mathrm{M}$ zeroes in horizontal and $\mathrm{N}$ zeroes in vertical directions.

Extension Function $E$ :

$\mathrm{f}_{\mathrm{r}}^{\mathrm{n}}=\mathrm{E}\left(\mathrm{f}_{\mathrm{r}-1}^{\mathrm{n}}\right)$

Construction of pyramid :

$$
f_{r+1}=P\left(f_{r}\right)
$$

$$
\text { lr } \square \text { fr } \square \mathrm{E} \text { (fr } \square 1 \text { ) }
$$

where $1_{0}, l_{1}, \ldots, l_{\mathrm{r}-1}$ are Laplacian image pyramids that contain band pass filtering images and keeping these records to utilize on reconstruction process and fr is the coarser level image. The $r$ levels of image pyramid are represented as $\mathrm{P}_{\mathrm{r}} \rightarrow\left\{\mathrm{f}_{\mathrm{r}}, \mathrm{l}_{0}, \mathrm{l}_{1}, \ldots, \mathrm{l}_{\mathrm{r}-1}\right\}$. At coarser level $\mathrm{f}_{\mathrm{r}}{ }_{\mathrm{r}}=$ $\mathrm{f}_{\mathrm{r}}$, since there is no more decomposition beyond this level. Reconstruction of the pyramid is done using:

$$
\mathrm{f}_{\mathrm{r}-1}^{n}=1_{\mathrm{r}-1}+\mathrm{E}\left(\mathrm{f}_{\mathrm{r}}^{\mathrm{r}}\right)
$$

Two images must be fused (Image1 \& Image2): for each image, the pyramid is constructed and the error records are preserved. Denote the constructed $r$ grades of Laplacian image pyramid for first and second image.

\section{2. Modified Principal Component Analysis (MPCA)}

After 2D LP-DCT based image fusion, a novel MPCA [16, 19, 31-32] method for obtaining the dimensionality reduction image is proposed and MPCA process is described in Algorithm 2. The detailed description of some key steps involved is as follows. 


\begin{tabular}{|c|c|}
\hline \multicolumn{2}{|c|}{ Algorithm 2. MPCA process } \\
\hline \multicolumn{2}{|c|}{ Input : Fused image by 2D LP-DCT. } \\
\hline Step 1 & : Load the fused image. \\
\hline Step 2 & : Compute $\mathrm{C}=\operatorname{cov}([\operatorname{im} 1(:)])$ \\
\hline Step 3 & : $[\mathrm{V}, \mathrm{D}]=\operatorname{eig}(\mathrm{C})$ \\
\hline Step 4 & $:[\max$, ind $]=\operatorname{sort}\left(\operatorname{diag}(\mathrm{D}),{ }^{\prime} \operatorname{descend} \mathrm{d}^{\prime}\right)$ \\
\hline Step 5 & $: \mathrm{a}=\mathrm{V}(:, \operatorname{ind}(1)) . / \operatorname{sum}(\mathrm{V}(:, \operatorname{ind}(1)))$ \\
\hline Step 6 & : F_E_img $=\mathrm{a}(1) *$ im 1 \\
\hline
\end{tabular}

The MPCA involves as mathematical procedure to transform correlated variables into number of uncorrelated principle components. It computes optimal description for a given data set with compact operation. The first principal of MPCA is to estimate covariance values for given set of data. Maximum variance is computed from the first principle component.

Let the source image is arranged as one column vector. The following steps are needed to project the data in 1D subspace.

- Arrange the data in a vector.

- Compute the covariance matrix for the given vector.

- Compute Eigen values for given covariance matrix.

- Find out V, D from the Eigen function.

- Sort the D in order of decreasing eigenvalue.

- Compute first column of V corresponds to larger Eigen value. To compute $\mathrm{P}$ as

$$
\begin{aligned}
& \mathrm{P}=\mathrm{V}(:, \text { ind }(1)) . / \operatorname{sum}(\mathrm{V}(:, \text { ind }(1)) \\
& \text { - Finally to get the features extracted image as } \\
& \mathrm{PCA}=\mathrm{P}(1) * \mathrm{Img}
\end{aligned}
$$

\section{RESULTS AND DISCUSSION}

Experiments were conducted on different standard multifocus image test pairs for image fusion research by online resource (www.imagefusion.org and www.mathworks.com). The fusion comparison only applies to five standardized test pairs: multifocus saras, flowerpot, clock, pepsi and disk. The fusion of images by a proposed method (2D LPDCT + MPCA) is compared with various types of methods: SWT, MSVD, NSCT, CV, DTCWT, RP, and LP. The comparison of performance is based on visual and quantitative measures for performance. All the source images are fused with 2D LPDCT and the dynamic range of an image with MPCA is increased.

Performance measures [30, 32, 33, 34, 35] such as $\mathrm{Q}^{\mathrm{AB} / \mathrm{F}}$ , QW, QS, QE, QC, QCB, CQM, QG, QM, QP, H, SD, SF, RMSE, PFE, MAE, SNR, PSNR, MSE, and PSNR_HVSM were evaluated for fused images of five sets of multifocus images. Table 1 and 2 gives the statistical details such as $\mathrm{Q}^{\mathrm{AB} / \mathrm{F}}, \mathrm{QW}, \mathrm{QS}, \mathrm{QE}, \mathrm{QC}, \mathrm{QCB}, \mathrm{CQM}, \mathrm{QG}, \mathrm{QM}, \mathrm{QP}, \mathrm{H}$, SD, SF, RMSE, PFE, MAE, SNR, PSNR, MSE, and PSNR_HVSM for the multifocus fused images. If parameters such as $\mathrm{Q}^{\mathrm{AB} / \mathrm{F}}, \mathrm{QW}, \mathrm{QS}, \mathrm{QE}, \mathrm{QC}, \mathrm{QCB}, \mathrm{CQM}$, QG, QM, QP, H, SD, SF, SNR, PSNR, and PSNR_HVSM are of greater value, the quality of the fused image will be improved while the other parameters (i.e. RMSE, PFE, MAE and MSE) should be of lower value. Since the aim of image fusion is to increase the information so as to make the fused image more appropriate for people's perceptions, visual analysis and quantitative analyses are necessary. Three criteria for visual analysis are frequently used in the literature: (1) transferring information from each individual picture to a fused image ; (2) lost information from the source pictures ; and (3) fused artifact.

The fused multifocus saras images derived through various fusion techniques are illustrated in Figure 1. The figure shows that, according to the proposed method, the image quality of the fused saras image ( Figure $1(\mathrm{j})$ ) is better than other methods without loss of information, and higher visual quality. It might be necessary, in comparisons to other fused images, to merge much of the information from both source images. The statistical parameters in Table 1 and 2 can be seen to quantitatively compare the results of the fused multifocus saras image. The proposed method has demonstrated good performance for all the statistical parameters for a multifocus saras image.

Visual examination of the Flowerpot images fused in Figure 2. Fused imagery can be observed by the proposed method ( Figure 2( j ) ), if compared to other fused images, with a large amount of data from both sources (Figure 2(a) and 2(b)). Table 1 and 2 shows that the performance of the proposed approach in terms of all parameters is superior to other methods.

Figure 3 Displays the visual comparison of the fused images by various methods in the multifocus clock. The fused image quality by the proposed approach ( Figure 3( j ) ) contains more information than the source and has the best visual effects. The resulting image quality is observed. Table 1 and 2 shows the performance criteria. The table showed that, in relation to all the statistical parameters, the proposed method is better than other methods expect RMSE, PFE, MAE, SNR, PSNR, MSE, and PSNR_HVSM.

The fused image of the multifocus pepsi by different methods shows visual performance comparison in Figure 4. Among the considered fusion methods, the proposed method ( Figure $4(\mathrm{j})$ ) has shown very good image quality in terms of all statistical parameters which was shown in table 1 and 2. In comparison to other algorithms, the statistical parameters achieved by the proposed method yield promising results expect MAE.

The images of disk and the results of different fusion based techniques are shown in Figure 5. The fused image derived from the proposed procedure has better visual quality than other methods based on fusion. Table 1 and 2 shows the statistical parameters of this multifocus image. The statistical parameters for the proposed method show better numerical values than compared to other techniques, according to this tables.

It is concluded from the results shown in these tables that the proposed algorithm provides better objective results as compared to other algorithms. The statistical results obtained for different multifocus images has shown that the proposed algorithm in this paper shows better performance than compared to other methods published recently elsewhere [24, 27, 31, 36, 37, 38]. 


\section{CONCLUSION}

2D Laplacian Pyramid-DCT (2D LP-DCT) with MPCA (Modified Principal Component Analysis) technique was proposed and applied to image fusion. Many more methods have been developed using DTCWT, NSCT, CV, SWT, MSVD, RP, LP and compared. In order to check the reliability of the images, different quality assurance approaches are evaluated. The proposed 2D LP-DCT with MPCA shows better performance assessment metrics, which in turn has better image quality without any information loss or objects, among the various techniques applied to different pairs of multifocus images.

\section{BIBLIOGRAPHY}

1. J. G. Liu, Smoothing filter-based intensity modulation: A spectral preserve image fusiontechnique for improving spatial details, Int. J. Remote Sensing, 21(18) (2000), 3461-3472.

2. M. Li, W. Cai, and Z. Tan, A region-based multi-sensor image fusion scheme using pulse-coupled neural network, Pattern Recognition Letters, 27 (2006), 19481956.

3. Zhan Kun, Jicai Teng, Qiaoqiao Li, and Jinhui Shi, A novel explicit multi-focus imagefusion method, J. Information Hiding and Multimedia Signal Processing, 6(3) (2015), 600-612.

4. Kun Zhan, Yuange Xie, Haibo Wang, and Yufang Min, Fast filtering image fusion, J.Electronic Imaging, 26(6) (2017), 063004.

5. Wen Li, Yuange Xie, Haole Zhou, Ying Han, and Kun Zhan, Structure-aware imagefusion, Optik - Int. J. for Light and Electron Optics, Elsevier, 172 (2018), 1-11.

6. C. Pohl, and J.L. Van Genderen, Multisensor image fusion in remote sensing: concepts, methods, and applications, Int. J. Remote Sensing, 19 (5) (1998), 823 854.

7. SusmithaVekkot, and Pancham Shukla, A Novel Architecture for Wavelet based Image Fusion, World Academy of Science, Engineering and Technology, 57 (2009), 372-377.

8. Gonzalo Pajares, and Jesus Manuel de la Cruz, A wavelet-based fusion tutorial, PatternRecognition, 37 (2004), 1855-1872.

9. Heng Ma, Chuanying Jia, and Shuang Liu, Multisource Image Fusion Based on WaveletTransform, Int. J. Information Technology, 11(7) (2005), 81-91.

10. Mark J. Shensa, The discrete wavelet transform: Wedding the trous and Mallatalgorithms, IEEE Trans. Signal Process, 40(10) (1992), 2464-2482.

11. Yufeng Zheng, Edward A. Essock, and Bruce C. Hansen, An advanced image fusionalgorithm based on wavelet transform: incorporation with PCA and morphologicalprocessing, Proc. SPIE-IS\&T Electronic Imaging, 5298 (2004), 177-187.

12. Shrivsubramani Krishnamoorthy, and K P Soman, Implementation and ComparativeStudy of Image Fusion Algorithms, Int. J. Computer Applications, 9(2) (2010), $0975-8887$.

13. Svante Wold, Principal Component Analysis, Chemometrics and Intelligent LaboratorySystems, Elsevier Science Publisher B.V, 2 (1987), 37-52.

14. C. Rama Mohan, Dr. S. Kiran, and R. Pradeep Kumar Reddy, Multi-focus ImageSynthesis based on DWT and Texture with Sharpening, Int. J. Information Technologyand Computer Science Perspectives, Pezzottaite Journals, 4(4) (2015), 1662-1670.

15. C. Rama Mohan, Dr. S. Kiran, and R. Pradeep Kumar Reddy, A Study on Several ImageSynthesis Algorithms,
Int. J. Information Technology and Computer Science Perspectives,Pezzottaite Journals, 4(3) (2015), 16001608.

16. VPS Naidu, and J.R. Raol, Fusion of Out Of Focus Images using Principal ComponentAnalysis and Spatial Frequency, J. Aerospace Sciences and Technologies, 60(3) (2008), 216-225.

17. H. Li, B. S. Manjunath, and S. K. Mitra, Multisensor image fusion using the wavelettransform, Graphical Models and Image Processing, 57(3) (1995), 235-245.

18. A. Toet, Image fusion by a ratio of low-pass pyramid, Pattern Recognition letters, 9(4) (1989), 245-253.

19. VPS Naidu, and J.R. Raol, Pixel-Level Image Fusion using Wavelets and PrincipalComponent Analysis - A Comparative Analysis, Defence Science Journal, 58(3) (2008),338-352.

20. Amaj Chamankar, Mansour Sheikhan, and Farhad Razaghian, Multi-Focus Image Fusion.Using Fuzzy Logic, IEEE, (2013).

21. . VPS Naidu, Discrete Cosine Transform based Image Fusion Techniques,J. Communication, Navigation and Signal Processing, 1(1) (2012), 35-45.

22. VPS Naidu, Block DCT based Image Fusion Techniques, e-Journal of Science andTechnology, 49-66.

23. Veerpal Kaur, and Jaspreet Kaur, Frequency Partioning Based Image Fusion for CCTV, Int. J. Computer Science and Information Technologies, 6 (4) (2015), 3968-3972.

24. Bin Yang, Jinying Zhong, Yuehua Li and Zhongze Chen, Multi-focus image fusion and super-resolution with convolutional neural network, International Journal of Wavelets, Multiresolution and Information Processing 15(4) (2017) 1750037-1 to 1750037-15.

25. - Deepak Gambhir, and Meenu Manchanda, Fusion of Color Images Based on Fuzzy Transform and Spatial Frequency, International Journal of Computational Intelligence and Applications 17(1) (2018) 1850004-1 to 1850004-9.

26. VPS Naidu, and Bindu Elias, A Novel Image Fusion Technique using DCT based Laplacian Pyramid, Int. J. Inventive Engineering and Sciences, 1(2) ( 2013).

27. VPS Naidu, Novel Image Fusion Techniques using DCT, Int. J. Computer Science andBusiness Informatics, 5(1) (2013).

28. Mohan C.R., and Kiran S. Image Enrichment Using Single Discrete Wavelet TransformMulti-resolution and Frequency Partition, Artificial Intelligence and EvolutionaryComputations in Engineering Systems, Advanced in Intelligent Systems and Computing,Springer, 668.

29. Svante Wold, Principal Component Analysis, Chemometrics and Intelligent LaboratorySystems, 2 (1987), 37-52.

30. P. Jagalingam, and A.V. Hegde, A Review of Quality Metrics for Fused Image, ElsevierTransaction, Aquatic Procedia, 4 (2015), 133-142.

31. B. K. Shreyamsha Kumar, Multifocus and multispectral image fusion based on pixelsignificance using discrete cosine harmonic wavelet transform, Springer-Verlag LondonLimited, (2012).

32. Betsy Samuel, and Vidya N, Full Reference Image Quality Assessment for BiometricDetection, Int. J. Modern Trends in Engineering and Research, 02 (06) (2015).

33. Mayuresh Gulame, K. R. Joshi, and Kamthe R. S, A Full Reference Based Objective ImageQuality Assessment, Int. J. Advanced Electrical and Electronics Engineering, 2(6) (2013). 
34. Ratchakit Sakuldee, and Somkait Udomhunsakul, Objective Performance of CompressedImage Quality Assessments, Int. J. Computer and Information Engineering, 1 (2007).

35. Pedram Mohammadi, Abbas Ebrahimi-Moghadam, and Shahram Shirani, Subjective andObjective Quality Assessment of Image: A Survey, Elsevier, (2014).

36. N. Radha, and T. Ranga Babu, Performance evaluation of quarter shift dual tree complex wavelet transform based multifocus image fusion using fusion rules, Int. J.

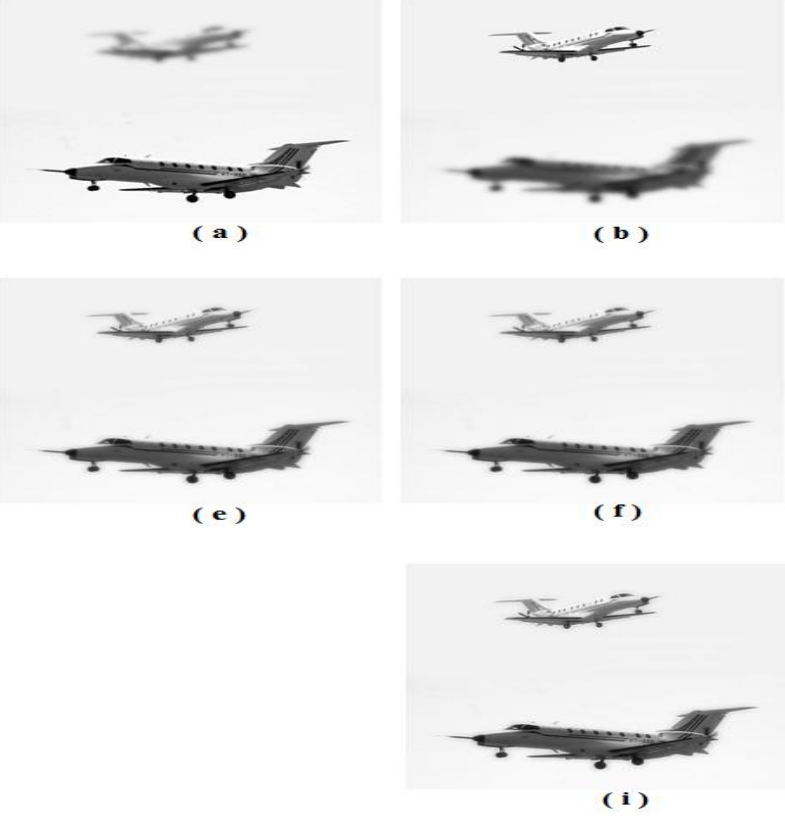

of Electrical and Computer Engineering (IJECE) 9(4) (2019), 2377-2385.

37. B. K. Shreyamsha Kumar, Image Fusion based on Pixel Significance using Cross Bilateral Filter, Signal, Image and Video Processing, 9(5) (2015), 1193-1204.

38. Yong Yang, Song Tong, Shuying Huang, and Pan Lin, Dual-Tree Complex Wavelet Transform and Image Block Residual-Based Multi-Focus Image Fusion in Visual Sensor Networks, Sensors, 14 (2014), 2240822430.
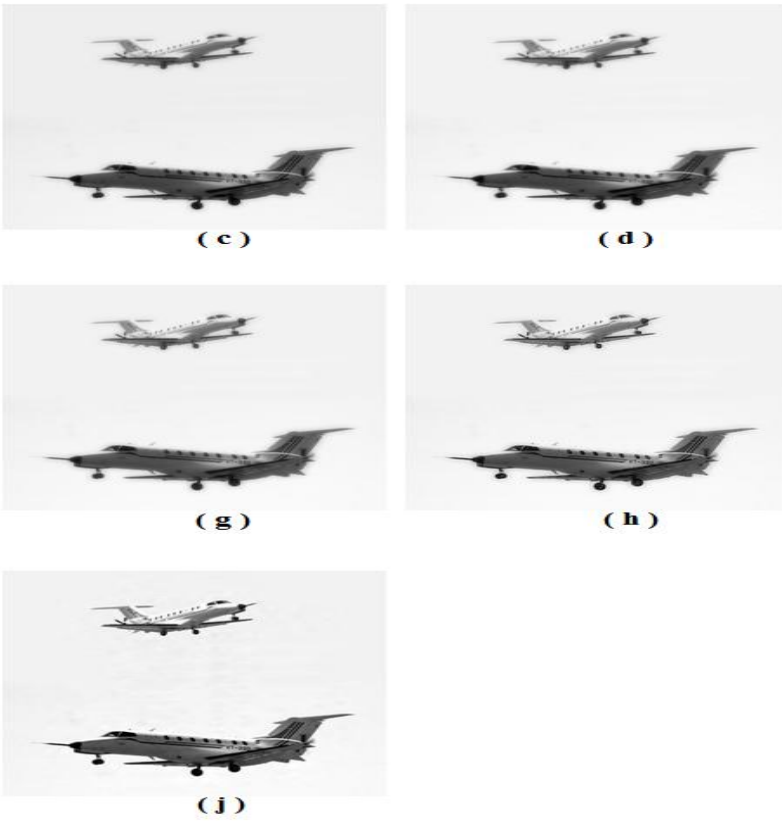

Figure 1: Fusion results of first pair of multifocus images (Saras): (a) input image (X), (b) input image (Y), (c) SWT, (d) MSVD, (e) NSCT, (f) CV, (g) DTCWT, (h) RP, (i) LP, (j) Proposed Method.
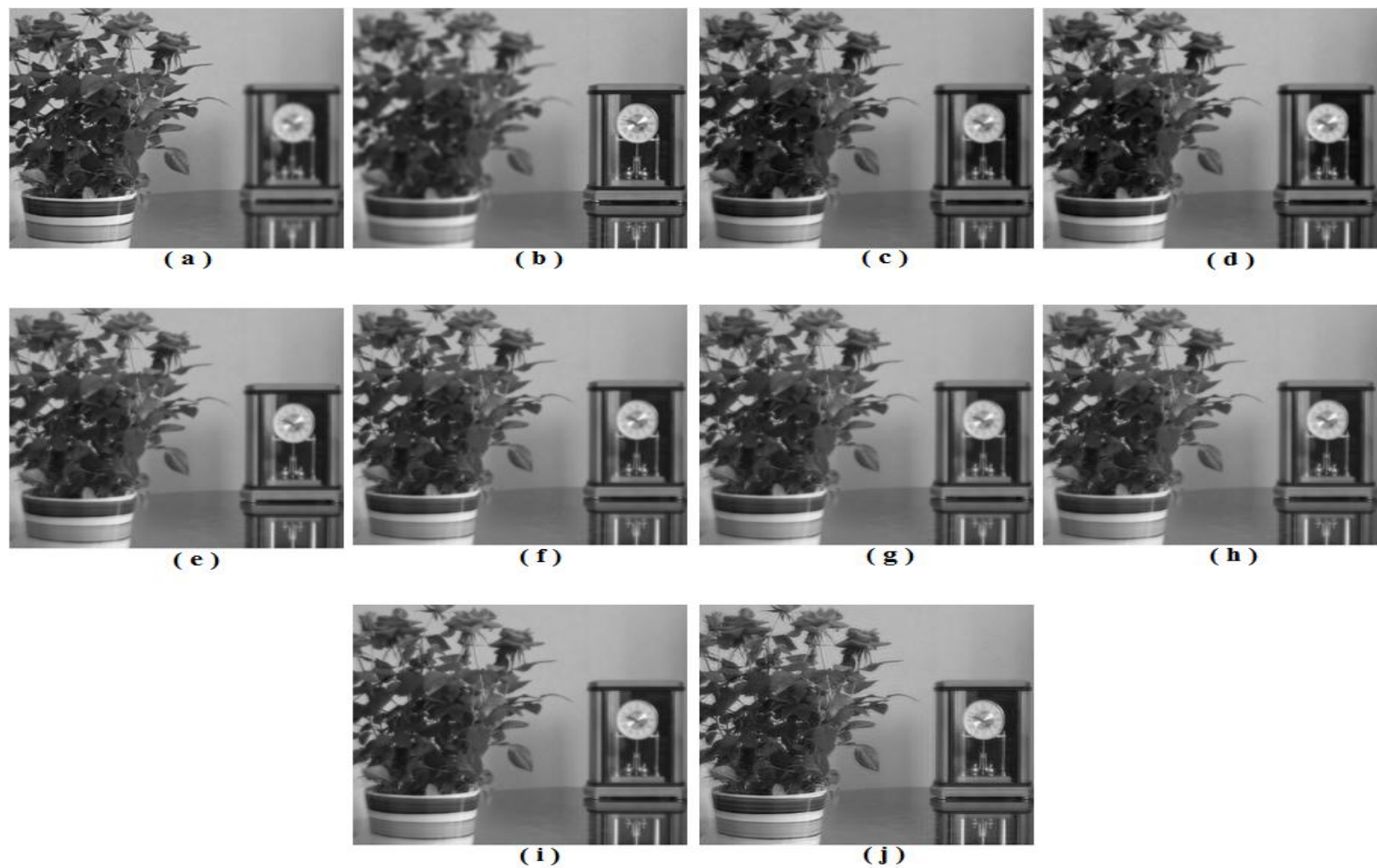

Figure 2: Fusion results of first pair of multifocus images (Flowerpot): (a) input image (X), (b) input image (Y), (c) SWT, (d) MSVD, (e) NSCT, (f) CV, (g) DTCWT, (h) RP, (i) LP, (j) Proposed Method.

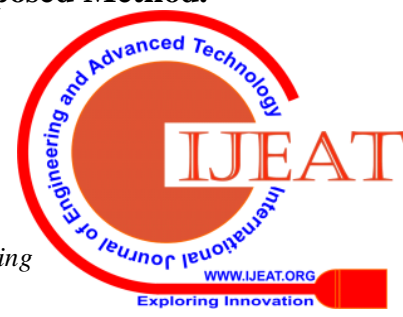



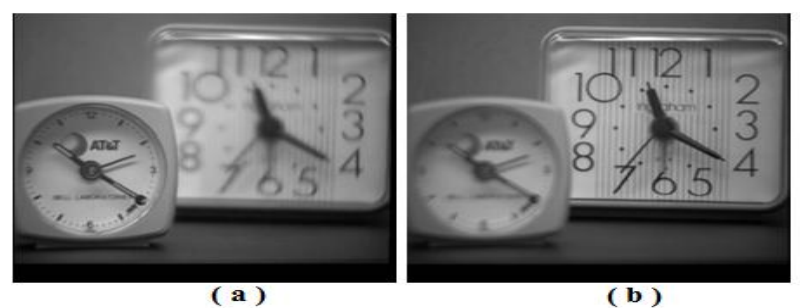

( b )

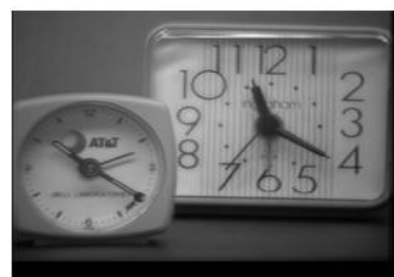

( e )

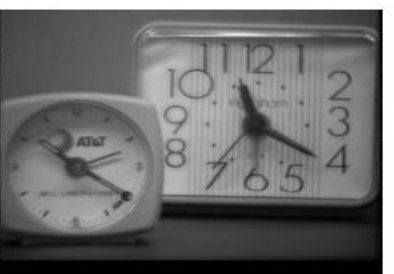

( f)

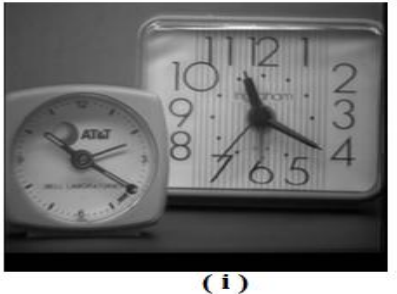

(i)

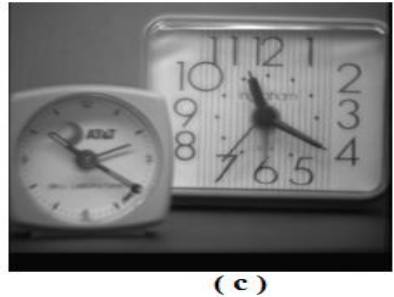

(c)

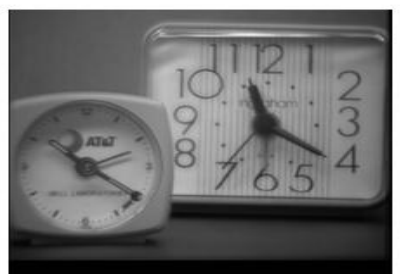

( g )

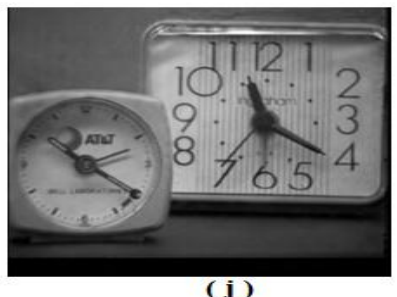

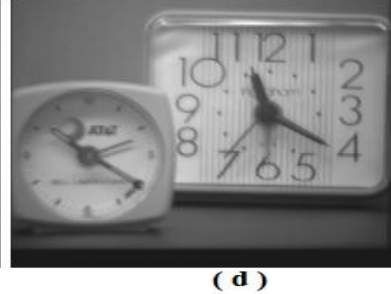

(d)

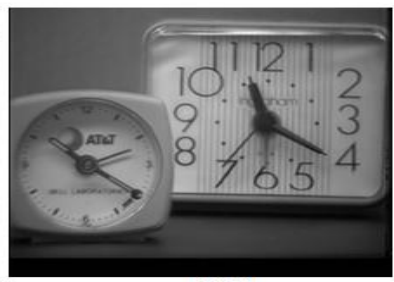

( h )

Figure 3: Fusion results of first pair of multifocus images (Clock): (a) input image (X), (b) input image (Y), (c) SWT, (d) MSVD, (e) NSCT, (f) CV, (g) DTCWT, (h) RP, (i) LP, (j) Proposed Method.

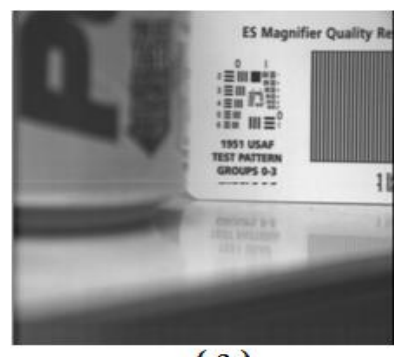

( a )

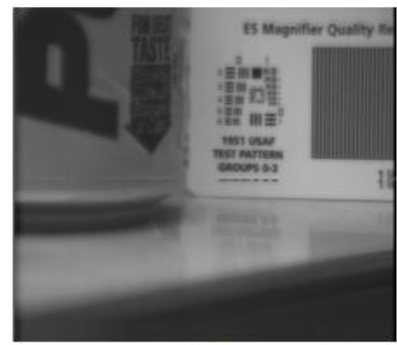

(e)

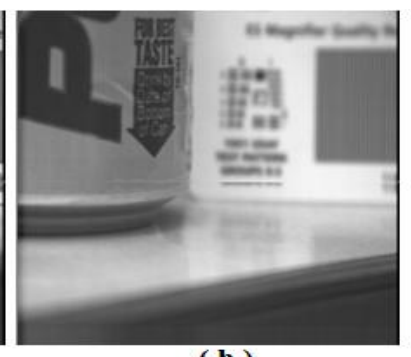

( b )

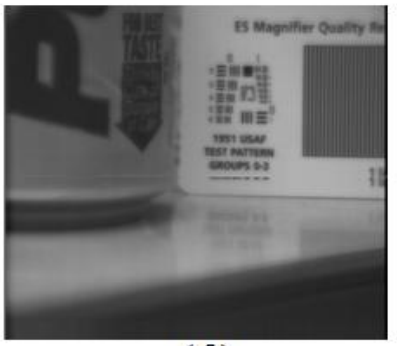

(f)

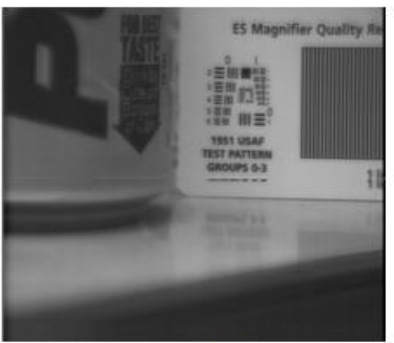

(i)

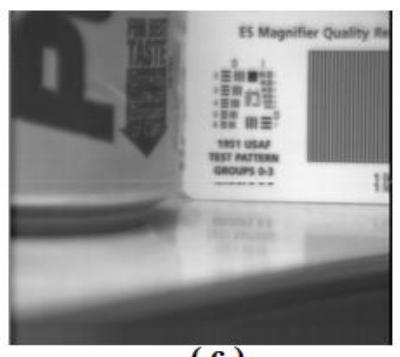

(c)

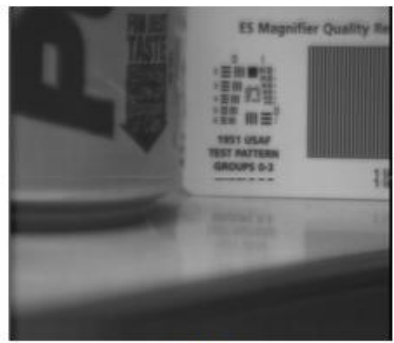

( g )

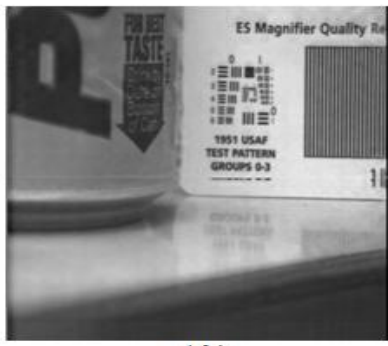

( j )

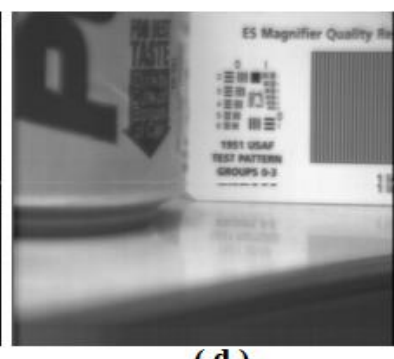

(d)

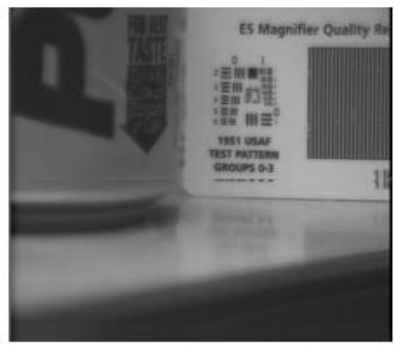

( h )

Figure 4: Fusion results of first pair of multifocus images (Pepsi): (a) input image (X), (b) input image (Y), (c) SWT, (d) MSVD, (e) NSCT, (f) CV, (g) DTCWT, (h) RP, (i) LP, (j) Proposed Method.

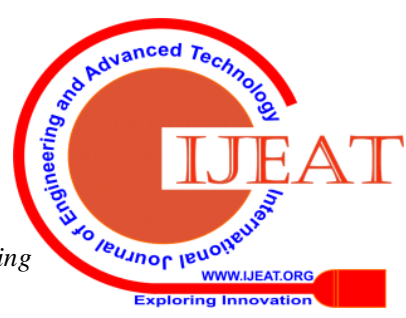




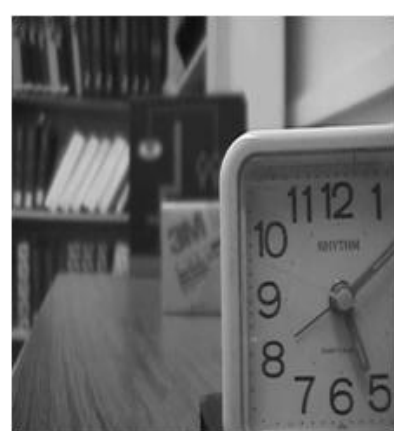

( a )

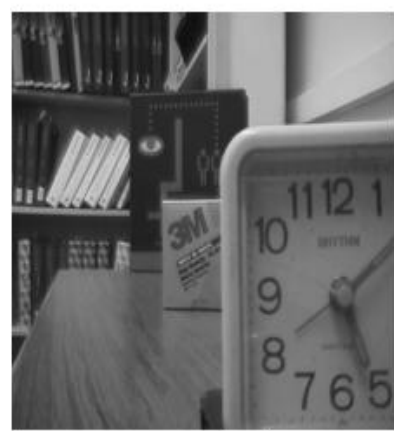

(e)

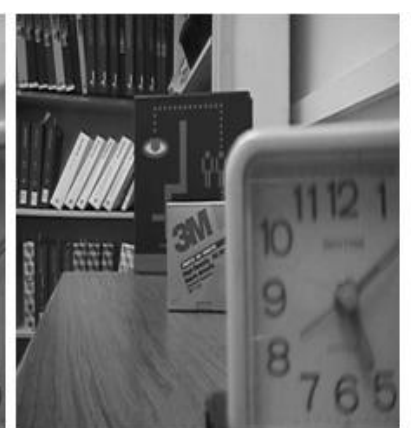

(b)

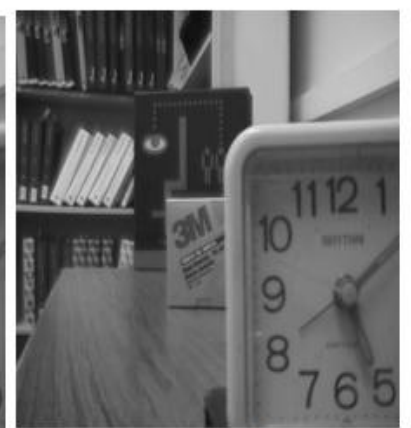

(f)

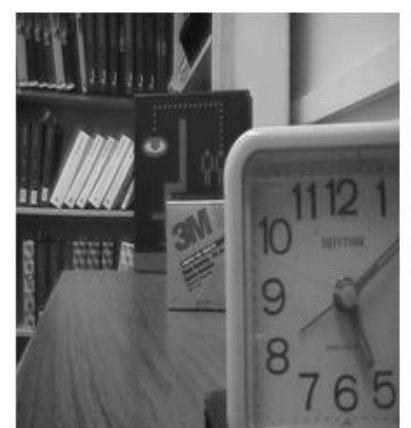

(i)

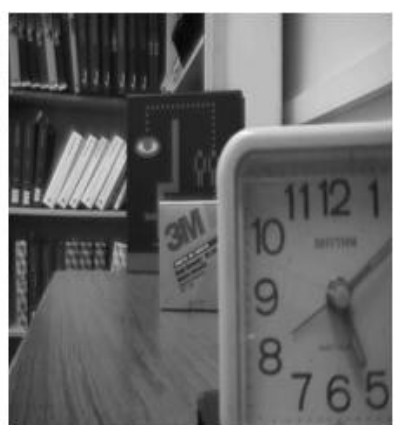

(c)

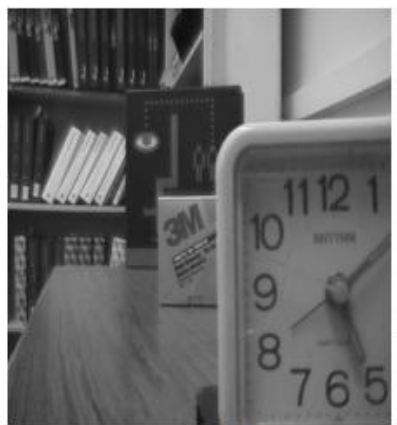

( g )

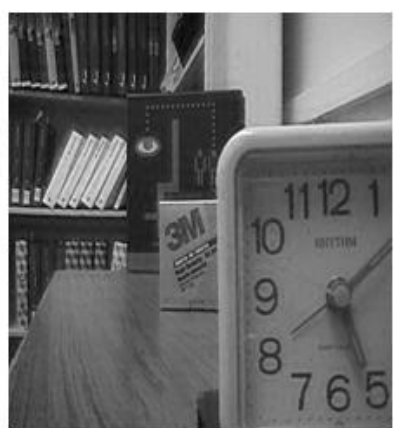

( j)

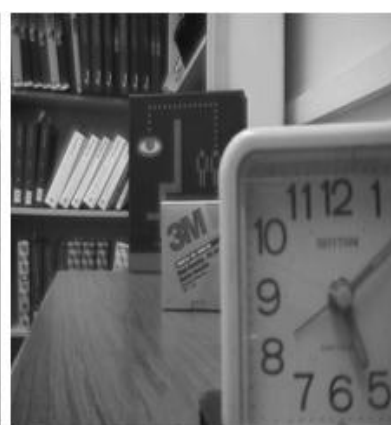

(d)

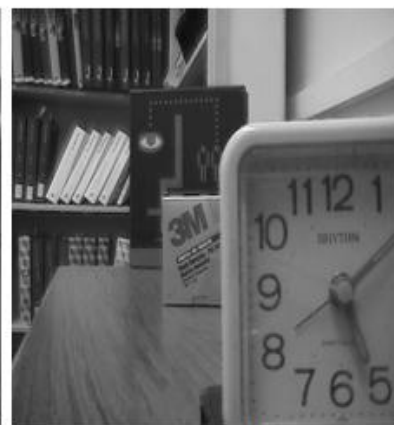

( h )

Figure 5: Fusion results of first pair of multifocus images (Disk): (a) input image (X), (b) input image (Y), (c) SWT, (d) MSVD, (e) NSCT, (f) CV, (g) DTCWT, (h) RP, (i) LP, (j) Proposed Method.

Table 2 Conventional Image Fusion Performance

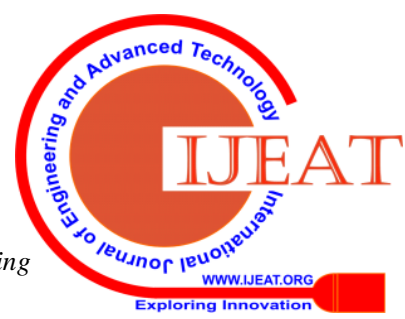


IMAGE ENHANCEMENT BASED ON FUSION USING 2D LPDCT AND MODIFIED PCA

Table 1 Obiective Image Fusion Performance

\begin{tabular}{|c|c|c|c|c|c|c|c|c|c|c|}
\hline Algorithm & $Q^{\mathrm{AB} / \mathrm{F}}$ & $\mathbf{Q}_{\mathrm{w}}$ & $Q_{s}$ & $Q_{E}$ & $\mathbf{Q}_{\mathrm{C}}$ & $\mathbf{Q}_{\mathrm{CB}}$ & $\mathrm{C}_{\mathrm{QM}}$ & $\mathbf{Q}_{\mathrm{G}}$ & $\mathbf{Q}_{M}$ & $Q_{\mathbf{P}}$ \\
\hline Input Image & Saras & & & & & & & & & \\
\hline SWT & 0.790545831 & 0.841502631 & 0.350404024 & 0.595085655 & 0.495307548 & 0.592443682 & 0.858954863 & 0.297209389 & 0.188704458 & 0.681787078 \\
\hline MSVD & 0.698777672 & 0.811437937 & 0.339322181 & 0.51104624 & 0.467873228 & 0.583976805 & 0.841034803 & 0.235132057 & 0.143070395 & 0.585581696 \\
\hline NSCT & 0.928375987 & 0.876270942 & 0.36360506 & 0.741240221 & 0.509951854 & 0.580743425 & 0.890303188 & 0.447013021 & 0.301137902 & 0.700255262 \\
\hline $\mathrm{CV}$ & 0.873173705 & 0.861444383 & 0.342896686 & 0.689157307 & 0.483140784 & 0.55939342 & 0.878118977 & 0.254072707 & 0.24938887 & 0.648373178 \\
\hline DTCWT & 0.925015639 & 0.874665134 & 0.361465395 & 0.732646599 & 0.507333615 & 0.603812679 & 0.888287567 & 0.453957045 & 0.283300131 & 0.697164307 \\
\hline RP & 0.966352934 & 0.911882401 & 0.364305289 & 0.847894027 & 0.52406033 & 0.604227752 & 0.931091536 & 0.490193204 & 0.436956025 & 0.694712599 \\
\hline LP & 0.98205488 & 0.916780516 & 0.36725848 & 0.87106685 & 0.530154092 & 0.62732486 & 0.929625506 & 0.500241401 & 0.524741714 & 0.737212208 \\
\hline $\begin{array}{l}\text { Proposed } \\
\text { Method }\end{array}$ & 0.999569966 & 0.958203872 & 0.527038169 & 0.952077096 & 0.947773904 & 0.870986091 & 0.962105321 & 0.891244858 & 2.630948298 & 0.784696548 \\
\hline Input Image & Flowerp & & & & & & & & & \\
\hline SWT & 0.865277278 & 0.861131918 & 0.792630041 & 0.662013735 & 0.793421267 & 0.537108531 & 0.881360828 & 0.466703441 & 0.938007676 & 0.696431123 \\
\hline MSVD & 0.646634495 & 0.794400017 & 0.675883813 & 0.388901632 & 0.684108546 & 0.525768552 & 0.844847409 & 0.348631416 & 0.510452677 & 0.414497538 \\
\hline NSCT & 0.903946856 & 0.869818461 & 0.795716219 & 0.710464118 & 0.800542045 & 0.531039045 & 0.890801124 & 0.480891928 & 0.995881759 & 0.69649286 \\
\hline $\mathrm{CV}$ & 0.86877314 & 0.86292288 & 0.792319007 & 0.681485587 & 0.792447621 & 0.531964666 & 0.885183044 & 0.452144704 & 0.917194343 & 0.663936969 \\
\hline DTCWT & 0.901062053 & 0.869104266 & 0.796285713 & 0.707008954 & 0.799326632 & 0.531410459 & 0.890320958 & 0.475033186 & 0.979732787 & 0.692982365 \\
\hline $\mathrm{RP}$ & 0.929652462 & 0.881433451 & 0.786455475 & 0.754409635 & 0.800858131 & 0.505774626 & 0.905442991 & 0.50532689 & 0.964673192 & 0.634209869 \\
\hline LP & 0.973292309 & 0.899807522 & 0.799849855 & 0.815015036 & 0.821806142 & 0.532918946 & 0.918110403 & 0.538150338 & 1.186178954 & 0.726800809 \\
\hline $\begin{array}{l}\text { Proposed } \\
\text { Method }\end{array}$ & 0.98346993 & 0.936116808 & 0.855368114 & 0.909433259 & 0.910235219 & 0.782143705 & 0.946769392 & 0.717098042 & 2.280334622 & 0.771762244 \\
\hline Input Image & Clock & & & & & & & & & \\
\hline SWT & 0.919927787 & 0.918751829 & 0.8752939 & 0.719866989 & 0.871417303 & 0.629540598 & 0.940226321 & 0.612552146 & 0.745034927 & 0.824256251 \\
\hline MSVD & 0.840414938 & 0.867759839 & 0.818294178 & 0.565512122 & 0.816826735 & 0.518675196 & 0.915788955 & 0.543614145 & 0.471968327 & 0.684677548 \\
\hline NSCT & 0.967609373 & 0.925475947 & 0.867428917 & 0.781999407 & 0.862559238 & 0.609062357 & 0.947179161 & 0.615784795 & 0.841558624 & 0.850866197 \\
\hline $\mathrm{CV}$ & 0.928106951 & 0.917000394 & 0.860938288 & 0.734118172 & 0.855654441 & 0.60037189 & 0.940192164 & 0.566222695 & 0.724413454 & 0.79480322 \\
\hline DTCWT & 0.966764143 & 0.924920319 & 0.867724873 & 0.776304 & 0.863078858 & 0.609759919 & 0.94664109 & 0.613527122 & 0.800535809 & 0.84697085 \\
\hline RP & 0.977487042 & 0.929633336 & 0.85993941 & 0.823965008 & 0.858646926 & 0.701377414 & 0.95357513 & 0.619430772 & 1.155105583 & 0.846419299 \\
\hline LP & 0.981548335 & 0.931711604 & 0.856137913 & 0.836118786 & 0.855979088 & 0.64942689 & 0.954194219 & 0.628544957 & 1.157888442 & 0.87258436 \\
\hline $\begin{array}{l}\text { Proposed } \\
\text { Method }\end{array}$ & 0.984618849 & 0.977522709 & 0.903010068 & 0.940765712 & 0.943666404 & 0.852514553 & 0.985707519 & 0.794880179 & 2.187290716 & 0.94155655 \\
\hline Input Image & Pepsi & & & & & & & & & \\
\hline SWT & 0.831849888 & 0.859607866 & 0.897015206 & 0.654913994 & 0.894830378 & 0.524520964 & 0.879583247 & 0.724873377 & 0.935084335 & 0.759421377 \\
\hline MSVD & 0.600928926 & 0.755039516 & 0.801227402 & 0.328997392 & 0.80167307 & 0.506260176 & 0.8266128 & 0.5666866943 & 0.704906694 & 0.447092864 \\
\hline NSCT & 0.926538702 & 0.882169536 & 0.893754223 & 0.720184772 & 0.892691609 & 0.507631078 & 0.901261851 & 0.724955528 & 1.166664274 & 0.775964855 \\
\hline Algorithm & $\mathbf{Q}^{\mathrm{AB} / \mathrm{F}}$ & $\mathbf{Q}_{\mathbf{w}}$ & Qs & $\mathbf{Q}_{\mathbf{E}}$ & $\mathbf{Q c}_{\mathrm{c}}$ & $\mathbf{Q}_{\mathrm{CB}}$ & $\mathrm{C}_{\mathrm{QM}}$ & $\mathbf{Q}_{\mathrm{G}}$ & $\mathbf{Q}_{\mathbf{M}}$ & $\mathbf{Q P}_{\mathbf{P}}$ \\
\hline $\mathrm{CV}$ & 0.801839059 & 0.853426463 & 0.889649564 & 0.642449092 & 0.887957041 & 0.483268034 & 0.874818244 & 0.711322082 & 1.040653043 & 0.748338336 \\
\hline DTCWT & 0.925501195 & 0.882081997 & 0.894637031 & 0.718676533 & 0.894137463 & 0.5000972 & 0.90126342 & 0.726580762 & 1.12418495 & 0.775548155 \\
\hline RP & 0.974676566 & 0.922709513 & 0.893040998 & 0.838835602 & 0.893654529 & 0.545740431 & 0.942709738 & 0.720774878 & 1.260219002 & 0.710850148 \\
\hline LP & 0.993605218 & 0.931639283 & 0.898024328 & 0.864726787 & 0.899258621 & 0.538042991 & 0.947690441 & 0.73273996 & 1.377042335 & 0.804948317 \\
\hline $\begin{array}{l}\text { Proposed } \\
\text { Method }\end{array}$ & 0.995264352 & 0.954741781 & 0.941540763 & 0.923791655 & 0.945134658 & 0.814666927 & 0.965056847 & 0.827034679 & 2.384022772 & 0.89257443 \\
\hline Input Image & Disk & & & & & & & & & \\
\hline SWT & 0.877333355 & 0.859939007 & 0.793658318 & 0.644531975 & 0.804353424 & 0.597256272 & 0.885229894 & 0.488670048 & 0.559918615 & 0.667084375 \\
\hline MSVD & 0.855921613 & 0.857094509 & 0.787964189 & 0.645284676 & 0.797992434 & 0.561885486 & 0.887944902 & 0.484498578 & 0.806232758 & 0.602709034 \\
\hline NSCT & 0.914994146 & 0.866161854 & 0.805252902 & 0.68269901 & 0.816325047 & 0.585733725 & 0.890566897 & 0.512296469 & 0.592364219 & 0.687685226 \\
\hline $\mathrm{CV}$ & 0.85438877 & 0.851883834 & 0.792021265 & 0.623311285 & 0.800608006 & 0.583337766 & 0.87850854 & 0.46626233 & 0.516705782 & 0.646076732 \\
\hline DTCWT & 0.909551735 & 0.864530059 & 0.802505221 & 0.674315913 & 0.813534646 & 0.586530637 & 0.889107721 & 0.507178205 & 0.571681246 & 0.68685724 \\
\hline $\mathrm{RP}$ & 0.951889703 & 0.887154683 & 0.803947686 & 0.7751705 & 0.827856873 & 0.577781 & 0.915145494 & 0.547359439 & 0.698113525 & 0.620228932 \\
\hline LP & 0.982313179 & 0.901796378 & 0.814068403 & 0.816271061 & 0.842755863 & 0.56925784 & 0.923562104 & 0.581332421 & 0.811326667 & 0.716425391 \\
\hline $\begin{array}{l}\text { Proposed } \\
\text { Method }\end{array}$ & 0.987628703 & 0.969332394 & 0.880011605 & 0.945993322 & 0.936691173 & 0.787464864 & 0.978075576 & 0.786843431 & 2.061081679 & 0.843459436 \\
\hline
\end{tabular}


Table 2 Conventional Image Fusion Performance

\begin{tabular}{|c|c|c|c|c|c|c|c|c|c|c|}
\hline Algorithm & H & SD & SF & RMSE & PFE & MAE & SNR & PSNR & MSE & PSNR_HVSM \\
\hline Input Image & Saras & & & & & & & & & \\
\hline SWT & 4.023908527 & 45.97411847 & 10.09537082 & 9.38836841 & 4.02759698 & 2.953417947 & 27.89907987 & 38.43889807 & 88.14146141 & 24.70785719 \\
\hline MSVD & 4.023078614 & 45.88722428 & 9.654955733 & 10.24177198 & 4.393705923 & 3.184269248 & 27.14338029 & 38.06104828 & 104.89389 & 24.41242674 \\
\hline NSCT & 4.021622961 & 46.18809146 & 12.75184215 & 8.440302401 & 3.620877981 & 2.730624141 & 28.8237222 & 38.90121924 & 71.23870461 & 25.00633637 \\
\hline $\mathrm{CV}$ & 4.018226745 & 46.09039722 & 12.43390731 & 8.927697395 & 3.829969756 & 2.866600787 & 28.33609311 & 38.65740469 & 79.70378078 & 24.63386372 \\
\hline DTCWT & 4.019900151 & 46.16336806 & 12.55034482 & 8.495928141 & 3.644741347 & 2.748401314 & 28.76666573 & 38.872691 & 72.18079499 & 24.97886676 \\
\hline $\mathrm{RP}$ & 4.019456739 & 47.65974228 & 16.67219819 & 6.613388426 & 2.837134429 & 2.02334489 & 30.94240172 & 39.960559 & 43.73690648 & 27.65643777 \\
\hline LP & 4.010296733 & 46.94667591 & 15.08386372 & 6.403172295 & 2.746952002 & 2.119643103 & 31.22297858 & 40.10084743 & 41.00061544 & 27.41951626 \\
\hline $\begin{array}{l}\text { Proposed } \\
\text { Method }\end{array}$ & 4.300190534 & 50.00383834 & 16.93923896 & 1.265849529 & 0.543047686 & 0.707965851 & 45.30324065 & 47.14097846 & 1.602375031 & 41.78163757 \\
\hline Input Image & Flowerpot & & & & & & & & & \\
\hline SWT & 7.358820072 & 49.91333209 & 9.753083608 & 8.393248959 & 6.687046161 & 4.825833004 & 23.49531358 & 38.92549825 & 70.44662809 & 24.7829707 \\
\hline MSVD & 7.354879094 & 49.56489319 & 8.536076505 & 10.96648914 & 8.737190978 & 5.901120127 & 21.17256343 & 37.76412318 & 120.26388 & 23.83647427 \\
\hline NSCT & 7.362583196 & 49.98706141 & 11.03675513 & 8.145491999 & 6.489653919 & 4.720033013 & 23.75556925 & 39.05562609 & 66.3490399 & 24.90553085 \\
\hline $\mathrm{CV}$ & 7.360904239 & 49.95590134 & 10.94491501 & 8.351487752 & 6.653774287 & 4.820447193 & 23.53863871 & 38.94716082 & 69.74734767 & 24.70747102 \\
\hline DTCWT & 7.362325198 & 49.98042156 & 10.98213995 & 8.159093915 & 6.5004908 & 4.722628572 & 23.74107704 & 39.04837999 & 66.57081352 & 24.8922652 \\
\hline $\mathrm{RP}$ & 7.368774936 & 50.24224547 & 12.16559075 & 7.612143839 & 6.064726244 & 4.322691033 & 24.34377596 & 39.34972944 & 57.94473383 & 25.70567202 \\
\hline LP & 7.372052601 & 50.43205133 & 12.11665494 & 6.720740274 & 5.354529655 & 3.989471314 & 25.42557344 & 39.89062818 & 45.16834983 & 26.76406928 \\
\hline $\begin{array}{l}\text { Proposed } \\
\text { Method }\end{array}$ & 7.410601683 & 50.95615134 & 13.10958417 & 6.167018441 & 4.913369923 & 3.985523558 & 26.17241074 & 40.26404684 & 38.03211646 & 27.78316688 \\
\hline Input Image & Clock & & & & & & & & & \\
\hline SWT & 7.294602336 & 49.3301733 & 11.31286866 & 6.567147258 & 6.001695636 & 3.644117889 & 24.43452065 & 39.99103176 & 43.17842029 & 27.93438924 \\
\hline MSVD & 7.277071554 & 49.2421494 & 13.29104934 & 8.463387743 & 7.73466397 & 4.73889818 & 22.23117099 & 38.88935692 & 71.99834858 & 27.43173343 \\
\hline NSCT & 7.315617313 & 49.50351572 & 13.0330403 & 5.661006654 & 5.173576531 & 3.190427296 & 25.72418244 & 40.63586265 & 32.04705621 & 28.77398925 \\
\hline $\mathrm{CV}$ & 7.340878321 & 49.41297204 & 12.46327879 & 6.348342131 & 5.801730305 & 3.594347033 & 24.72884927 & 40.13819607 & 40.53391304 & 27.93062609 \\
\hline DTCWT & 7.299516253 & 49.48406105 & 12.87871069 & 5.681729411 & 5.192514995 & 3.194822952 & 25.69244481 & 40.61999384 & 32.2820764 & 28.76304583 \\
\hline RP & 7.305442734 & 50.01531871 & 15.96107847 & 4.966931586 & 4.539263466 & 2.454170765 & 26.86029219 & 41.20391753 & 24.67040938 & 30.66666895 \\
\hline LP & 7.322395092 & 50.13367818 & 15.2837938 & 4.18483941 & 3.824511838 & 2.263475406 & 28.34847981 & 41.94801134 & 17.66817934 & 31.54759892 \\
\hline $\begin{array}{l}\text { Proposed } \\
\text { Method }\end{array}$ & 7.362544196 & 52.02212829 & 16.55700454 & 5.923987502 & 5.413913918 & 3.663391113 & 25.32977308 & 40.43865797 & 35.09362793 & 28.16395535 \\
\hline Input Image & Pepsi & & & & & & & & & \\
\hline SWT & 97.57662563 & 44.00633572 & 10.68514921 & 5.564619711 & 5.171936008 & 2.684991495 & 25.72693715 & 40.71044441 & 30.96499253 & 28.38885921 \\
\hline Algorithm & H & SD & SF & RMSE & PFE & MAE & SNR & PSNR & MSE & PSNR_HVSM \\
\hline MSVD & 97.5767579 & 43.65395849 & 7.443489446 & 8.450150922 & 7.853841248 & 3.957128299 & 22.09835763 & 38.89615465 & 71.4050506 & 26.77211706 \\
\hline NSCT & 97.57704163 & 44.1015939 & 11.44601476 & 5.109624861 & 4.749049203 & 2.591952557 & 26.46786662 & 41.08090914 & 26.10859008 & 29.13921972 \\
\hline $\mathrm{CV}$ & 97.57704163 & 44.01947133 & 11.07205175 & 5.685989894 & 5.284741347 & 2.789473574 & 25.53952527 & 40.61673847 & 32.33106354 & 28.14786083 \\
\hline DTCWT & 97.57704163 & 44.09708003 & 11.39477113 & 5.111583838 & 4.750869939 & 2.570903428 & 26.46453718 & 41.07924442 & 26.12863958 & 29.1425535 \\
\hline RP & 97.6590023 & 44.49203703 & 12.95214883 & 3.784052373 & 3.517019623 & 1.965768303 & 29.07650417 & 42.38522792 & 14.31905236 & 32.34755267 \\
\hline LP & 97.59282735 & 44.52122483 & 12.86725306 & 3.394757308 & 3.155196306 & 1.869426938 & 30.0194723 & 42.85671199 & 11.52508384 & 33.15249634 \\
\hline $\begin{array}{l}\text { Proposed } \\
\text { Method }\end{array}$ & 97.74516525 & 46.02906702 & 14.14139463 & 3.098813624 & 2.880136755 & 2.144973755 & 30.81173781 & 43.25284474 & 9.602645874 & 34.65955453 \\
\hline Input Image & Disk & & & & & & & & & \\
\hline SWT & 7.20052667 & 44.22685704 & 10.29597226 & 8.253560941 & 7.605839778 & 4.460340453 & 22.37705655 & 38.99838568 & 68.1212682 & 25.15927928 \\
\hline MSVD & 7.204617595 & 44.34168646 & 11.14253716 & 8.319586495 & 7.666683793 & 4.521952552 & 22.30784897 & 38.96378189 & 69.2156279 & 25.25750169 \\
\hline NSCT & 7.20622778 & 44.29759543 & 11.16809597 & 8.010697625 & 7.382035837 & 4.313656894 & 22.63647702 & 39.12809592 & 64.17127644 & 25.28249899 \\
\hline $\mathrm{CV}$ & 7.203616098 & 44.18472807 & 10.63463957 & 8.553263052 & 7.882021932 & 4.549680303 & 22.06724722 & 38.84348102 & 73.15830884 & 24.8356384 \\
\hline DTCWT & 7.204733865 & 44.2751568 & 10.99961975 & 8.064273698 & 7.431407378 & 4.325345249 & 22.57857861 & 39.09914672 & 65.03251027 & 25.25014117 \\
\hline RP & 7.249232797 & 45.06131751 & 14.10797201 & 6.628000059 & 6.10784931 & 3.532433973 & 24.28223372 & 39.95097427 & 43.93038479 & 27.32419819 \\
\hline
\end{tabular}


IMAGE ENHANCEMENT BASED ON FUSION USING 2D LPDCT AND MODIFIED PCA

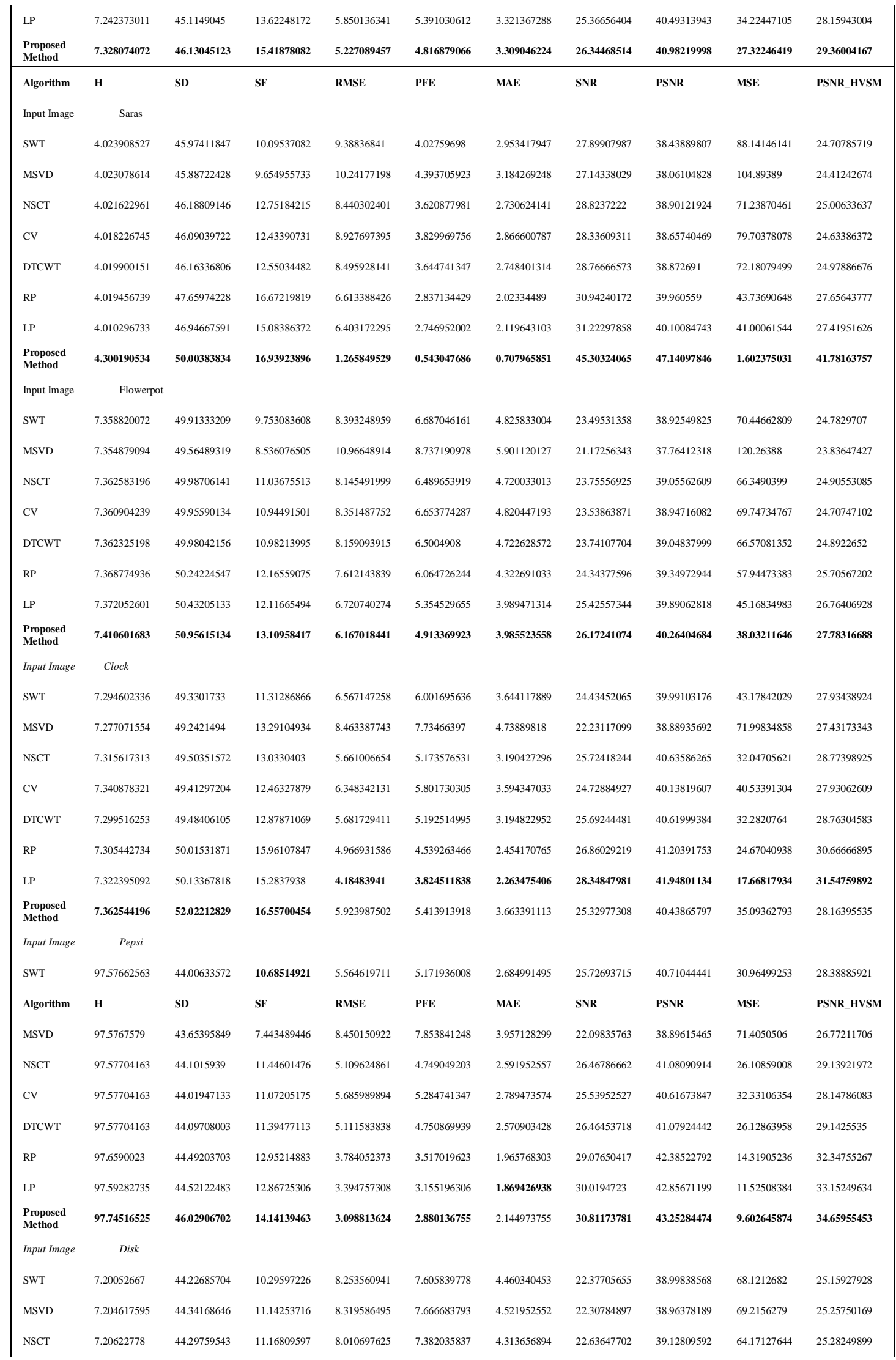




\begin{tabular}{|lllllllllll|} 
CV & 7.203616098 & 44.18472807 & 10.63463957 & 8.553263052 & 7.882021932 & 4.549680303 & 22.06724722 & 38.84348102 & 73.15830884 & 24.8356384 \\
DTCWT & 7.204733865 & 44.2751568 & 10.99961975 & 8.064273698 & 7.431407378 & 4.325345249 & 22.57857861 & 39.09914672 & 65.03251027 & 25.25014117 \\
RP & 7.249232797 & 45.06131751 & 14.10797201 & 6.628000059 & 6.10784931 & 3.532433973 & 24.28223372 & 39.95097427 & 43.93038479 & 27.32419819 \\
LP & 7.242373011 & 45.1149045 & 13.62248172 & 5.850136341 & 5.391030612 & 3.321367288 & 25.36656404 & 40.49313943 & 34.22447105 & 28.15943004 \\
$\begin{array}{l}\text { Proposed } \\
\text { Method }\end{array}$ & $\mathbf{7 . 3 2 8 0 7 4 0 7 2}$ & $\mathbf{4 6 . 1 3 0 4 5 1 2 3}$ & $\mathbf{1 5 . 4 1 8 7 8 0 8 2}$ & $\mathbf{5 . 2 2 7 0 8 9 4 5 7}$ & $\mathbf{4 . 8 1 6 8 7 9 0 6 6}$ & $\mathbf{3 . 3 0 9 0 4 6 2 2 4}$ & $\mathbf{2 6 . 3 4 4 6 8 5 1 4}$ & $\mathbf{4 0 . 9 8 2 1 9 9 9 8}$ & $\mathbf{2 7 . 3 2 2 4 6 4 1 9}$ & $\mathbf{2 9 . 3 6 0 0 4 1 6 7}$ \\
\hline
\end{tabular}

